\title{
Renal and red marrow dosimetry in peptide receptor radionu- clide therapy: 20 years of history and ahead.
}

\author{
Stephan Walrand ${ }^{1, *}$ and François Jamar ${ }^{2}$ \\ 1 Cliniques universitaires Saint-Luc, Brussels, Belgium. stephan.walrand@uclouvain.be \\ 2 Cliniques universitaires Saint-Luc, Brussels, Belgium. francois.jamar@uclouvain.be \\ * Correspondence: stephan.walrand@uclouvain.be
}

\begin{abstract}
The development of dosimetry and studies in peptide receptor radionuclide therapy (PRRT) over these two last decades are reviewed. Differences in kidney and bone marrow toxicity reported between ${ }^{90} \mathrm{Y},{ }^{177} \mathrm{Lu}$ and external beam radiotherapy (EBRT) are discussed with regards to the physical properties of these beta emitter radionuclides. The impact of these properties on the response to small and large tumors is also considered. Capacities of the imaging modalities to assess the dosimetry to target tissues are evaluated. Studies published in the last two years that confirm a red marrow uptake in ${ }^{177} \mathrm{Lu}$-DOTATATE therapy, as already observed 20 years ago in ${ }^{86} \mathrm{Y}$-DOTATOC PET studies, are commented and analyzed in the light of the recent knowledges in transferrin transport mechanism. The review enlightens the importance i) of using state of the art imaging modalities, ii) of individualizing the activity to be injected with regards to the huge tissue uptake variability observed between patients, iii) of challenging the currently used but inappropriate blood based red marrow dosimetry and iv) of considering individually tandem therapy. Last, a smart individually optimized tandem therapy taking benefit of the bi-orthogonal toxicity-response pattern of ${ }^{177} \mathrm{Lu}$-DOTATATE and of ${ }^{90} \mathrm{Y}$-DOTATOC is proposed.
\end{abstract}

Keywords: somatostatin, PRRT, dosimetry, DOTATOC, DOTATATE

\section{Introduction}

Peptide receptor radionuclide therapy (PRRT) is a well-established therapy for metastatic cancers expressing somatostatin receptors. A somatostatin analog peptide, such as octreotide or octreotate, is covalently bounded to a chelator, such as DTPA or DOTA. This chelator can be viewed as an empty basket. Before intravenous injection, a radionuclide $\left({ }^{111} \mathrm{In},{ }^{90} \mathrm{Y},{ }^{177} \mathrm{Lu}\right)$ is mixed with the chelator-peptide in a solution at a low $\mathrm{pH}$ and at a temperature both optimized to promote the entrance of the radionuclide within the chelator cage. High labelling fractions are easily reached, i.e. less than $2 \%$ of residual free radionuclide. The labeled compound exhibits a rather high stability when the $\mathrm{pH}$ solution is increased above 6 .

The quantity to be injected is expressed in becquerel $(\mathrm{Bq})$ which corresponds to one decay per second and is named activity. By analogy with pharmaceutical drug, this quantity is often named dose, which is incorrect: the term dose identifies the quantity of energy deposed in a tissue by the particles emitted during the decays. This dose is expressed in gray (Gy) corresponding to 1 joule $(\mathrm{J})$ delivered in $1 \mathrm{~kg}$ of tissue.

The first PRRT trials used ${ }^{11}$ In-DTPA-octreotide [1], a tracer initially developed for diagnostic intent. Due to the cell internalization of the tracer, the short range ${ }^{111}$ In auger electrons were considered suitable for tumors therapy [2]. The observed tumors control appeared promising, however an escalating activity study quickly revealed hematological concerns [3]. Indeed, as most of the ${ }^{111}$ In decay energy is converted into gamma rays, the cross-irradiation of the red marrow from the remainder of the body was significant [4].

This issue was overcome using ${ }^{90}$ Y-DOTA-octreotide ( ${ }^{90} \mathrm{Y}$-DOTATOC) [5], which for therapy intent can be considered as a pure beta emitter. This was confirmed by the ${ }^{90} \mathrm{Y}$ - 
DOTATOC clinical phase I trial SMT-487 [6,7]. With this compound, the first organ at risk was no longer the red marrow, but the kidneys, which is less life threatening [8]. However, due to its long beta range, i.e., maximal $11 \mathrm{~mm}$ in water, the dose delivered to small tumors is limited. Thus, relapse of micro-metastases initially not visible on imaging modalities were observed, although all known metastases completely responded.

To improve small tumor control, ${ }^{177} \mathrm{Lu}$-DOTA-octreotate (177 Lu-DOTATATE) was developed [9], the beta of which having a smaller range, i.e., $2 \mathrm{~mm}$ in water. Although the decay energy is mostly brought by the beta, the main tissue at risk identified in the phase III study was the red marrow, with about $10 \%$ of the patients undergoing grade 3 or 4 lymphopenia [10].

The aim of this paper is to provide a comprehensive and commented review of the literature evidencing and explaining the different toxicity and response patterns observed between ${ }^{90}$ Y-DOTATOC and ${ }^{177}$ Lu-DOTATATE PRRT. Last, by combining therapy improvements reported in the literature, a smart optimized tandem therapy design is proposed.

\section{Kidney dosimetry and toxicity}

The ${ }^{90}$ Y-DOTATOC clinical phase I trial ${ }^{86}$ Y-SMT-487 [6-8] was designed following the FDA requirements. The total injected activity was computed to deliver a maximum dose of 27 Gy to the kidneys, based on a pre-therapy ${ }^{86}$ Y-DOTATOC PET using the MIRD pamphlet no. 11 [11] which assumed a standard kidney volume for the dosimetry calculation. The PET scan was reconstructed using a dedicated prompt single gamma rays correction [12].

The trial included a cycle activity escalation, i.e., a reduction of the therapy cycles number needed to reach $27 \mathrm{~Gy}$ to the kidneys, starting from 5 cycles to a single cycle. The patient kidney follow-up was set as 5 years, with patients exhibiting a creatinine clearance lost per year up to $60 \%$. Fig. 1A clearly shows that obviously no toxicity-dose correlation was observed as all the patients, beside one getting an extra cycle for compassionate use, got the same kidney dose.

Rather than to conclude that dosimetry was useless, which sometimes happen in nuclear medicine, the patient $\mathrm{CT}$ analogic films were scanned and the renal volume measured: at this time, no hybrid SPECT-CT or PET-CT was available, and due to limited data storage capacity, most patient CT slices were only analogically archived. Fig. 1B shows that by just rescaling the kidney dose with the standard to individual kidney volume ratio, a toxicity dose relation became visible.
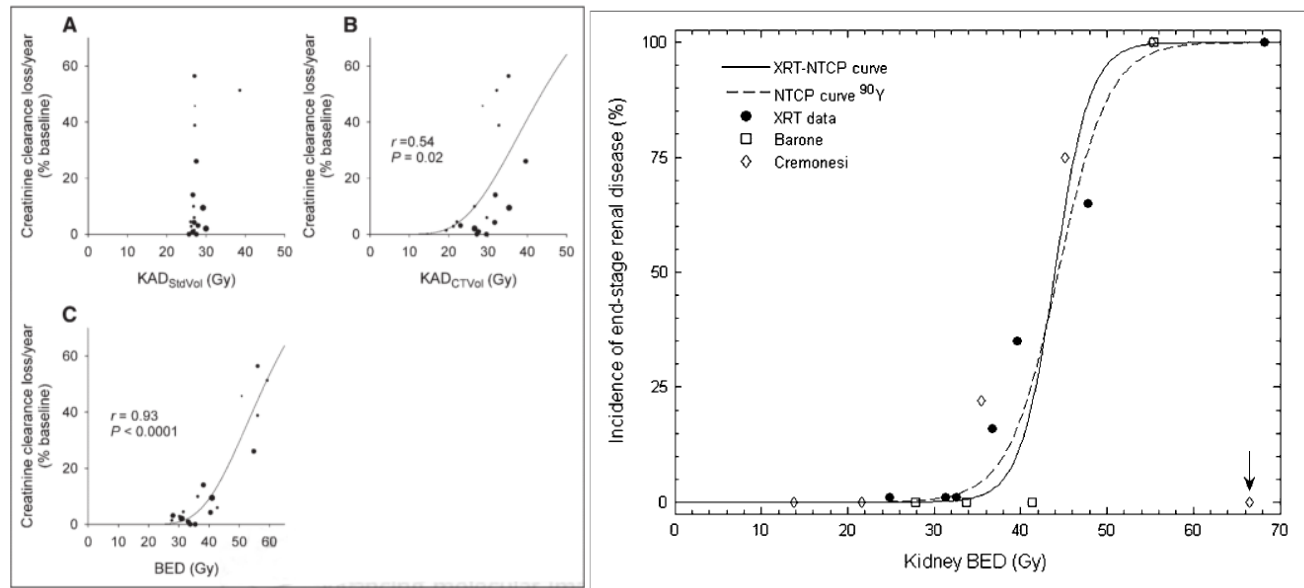

Figure 1: A: toxicity observed in the ${ }^{86} \mathrm{Y}-\mathrm{SMT}-487$ phase I trial as a function of the absorbed dose computed with the MIRD pamphlet no. 11 formalism [11], B: after rescaling with the individual kidney volume and C: converted into BED. The dots diameter corresponds to the cycles number. 
Reprinted from [8] with permission of the SNM. D: Matching of the NTCPs observed in ${ }^{90}$ Y-DOTATOC with that of EBRT. Reprinted from [13] with permission of the SNM.

It was noted that globally the patients receiving less cycles number underwent higher creatinine clearance lost per year (fig 1B). The absorbed doses were thus converted into a biological effective dose (BED) using the Lea-Catcheside formalism [14], and a clear toxicity-dose correlation appeared (fig. 1C). This toxicity-dose relation observed in nuclear medicine was the first one matching that observed in EBRT (fig. 1D): the dose calculation based on fractionation commonly used in EBRT was proved applicable in nuclear medicine.

In all PRRT studies, i.e., ${ }^{111}$ In-DTPA-octreotide SPECT [15], ${ }^{86}$ Y-DOTATOC PET [12] and ${ }^{177} \mathrm{Lu}$-DOTATATE SPECT [16] showed that the kidney uptake is localized in the cortex such as initially observed by Hammond et al. in planar ${ }^{111}$ In-DTPA-octreotide scintigraphy [17]. Intra-patient ${ }^{86} Y$-DOTATOC PET studies proved that amino-acid infusion significantly reduced the renal reuptake [6]. By competing with the megalin/cubilin complex on the apical membrane of proximal tubular cells (PTCs), basic amino acids, such as L-lysine and L-arginine can reduce by $\approx 50 \%$ the reuptake of the radiolabeled peptide. The other fraction is taken up by the PTCs by fluid-phase endocytosis, that is not influenced by the presence of high amounts of basic amino acids [18,19]. Studies in knock-out rat provided evidence that megalin is essential for renal tubule reabsorption of the peptide [20].

Ex vivo autoradiography of healthy kidney tissue, from patients who received ${ }^{111} \mathrm{In}-$ DTPA-octreotide before nephrectomy (fig. 2A), showed an uptake gradient decreasing from the inner to the outer cortex boundary [21]. As the radiosensitive glomerulus is about $6 \mathrm{~mm}$ far from the inner boundary (fig. 2E), its crossfire irradiation by the activity taken up by the tubules will strongly depend on the radionuclide beta range, as clearly showed in the Monte-Carlo (MC) isodose simulations (fig. 2 B-C). These MC simulations explain why the first limiting tissue is the kidney with ${ }^{90} \mathrm{Y}$-DOTATOC and the red marrow in ${ }^{177} \mathrm{Lu}$-DOTATATE PRRT, respectively (table 1).

Ex vivo autoradiography,
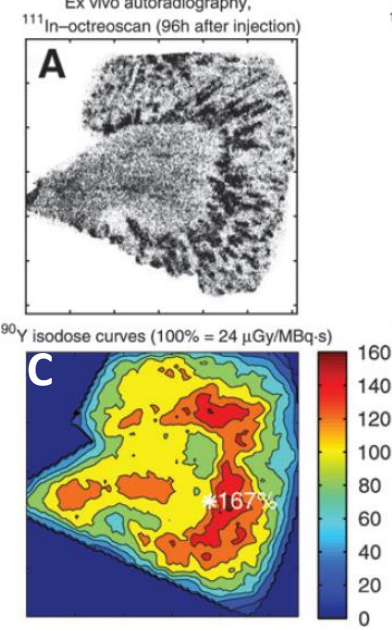

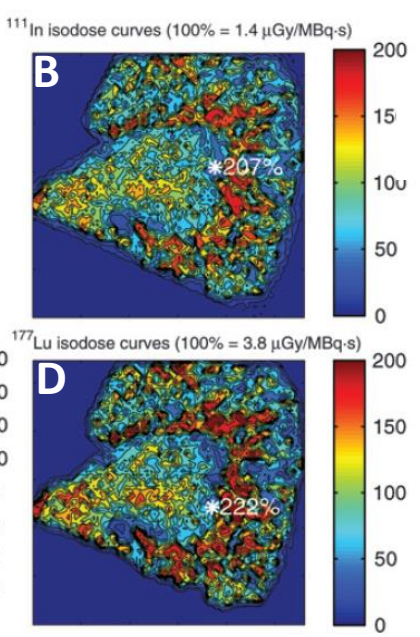

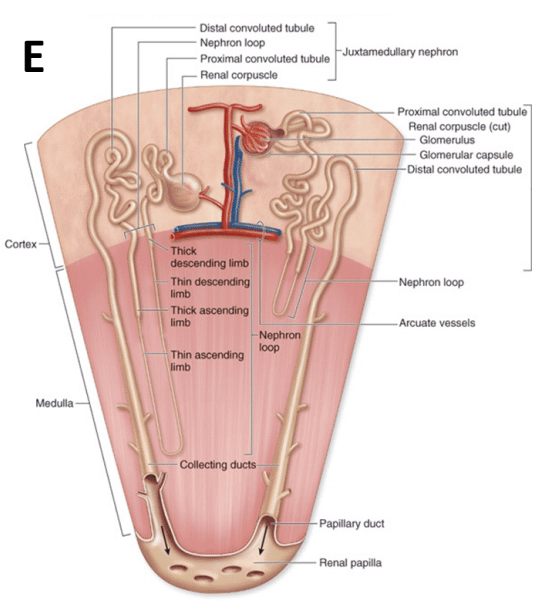

Figure 2: A: ${ }^{111}$ In autoradiography. B, C, D: corresponding isodoses simulated by Monte Carlo for ${ }^{111} \mathrm{In},{ }^{90} \mathrm{Y}$ and ${ }^{177} \mathrm{Lu}$, respectively. Reprinted from [21] with permission of the SNM. E: nephron anatomy showing the glomerulus and tubule location. Reprinted from [22] with permission of ....

\section{Red marrow dosimetry and toxicity}

A highly variable red marrow inter-patient uptake in PRRT was evidenced early in 2005 during the ${ }^{86}$ Y-DOTATOC phase I ${ }^{86}$ Y-SMT-487 trial which used state of the art PET imaging [23]. Furthermore, this red marrow uptake was intra-patient correlated to that measured with ${ }^{111}$ In-DTPA-octreotide by SPECT [24]. This was overlooked by the nuclear 
medicine community arguing that RM uptake was no visible in ${ }^{90} \mathrm{Y}$ bremsstrahlung or ${ }^{177} \mathrm{Lu}$ SPECT $[25,26]$. The case appeared to be solved in 2017 when the LutatheraTM insert package, which states that establishing red marrow dosimetry is useless in predicting observed toxicity, was agreed by the FDA and by the EMA [27].

These last years, several teams using state of the art SPECT/CT demonstrated clear red marrow uptake of ${ }^{177} \mathrm{Lu}$ in ${ }^{177} \mathrm{Lu}$-DOTATATE therapy [16], the dosimetry based on which enabling some toxicity prediction [28-30]. The observed red marrow dosimetry was about fourfold that of the blood-based method used in the Netter study [10], explaining why about 10\% Grade 3-4 hematological toxicity was observed in this study.

Such red marrow uptake could appear surprising with regards to the high DOTA affinity and stability for yttrium and for lutetium. However, in the transchelation competition with transferrin, DOTA is just a passive and naïve chelator stroke by an active and cunning thief. Iron is a vital compound for mammalians and evolution spent hundreds of millions of years to improve transferrin, versus a few decades for chemists.

Transferrin is a protein having two active lobes (fig. 3A) [31]. In the iron free state, i.e., apo-transferrin, the $\mathrm{N}$-lobe and the $\mathrm{C}$-lobe are open, ready to catch a metallic ion. When a metallic ion enters a lobe, the lobe closes and surrounds the ion as a result of the Van der Waals forces. In this state, no other external force can easily remove the ion from the locked lobe. When an appropriate anion binds to the corresponding active site, the lobe opens and releases the metallic ion. This key-padlock mechanism ensures that iron will be released to the appropriate cells and not elsewhere in the body.

Not only the ion transferrin release is an active process, but also the ion catching one. Inorganic chelators, such as DOTA, have to wait for the release of a metal ion by another chelator in order to trap it, which is rare from a good competitor. In contrary, kinetics and

A

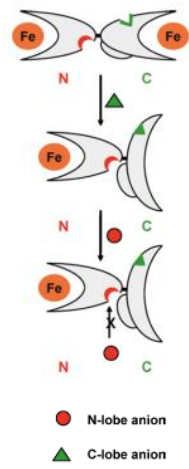

B

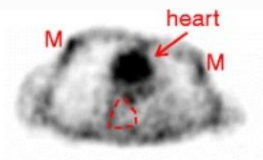

$4 \mathrm{~h}$

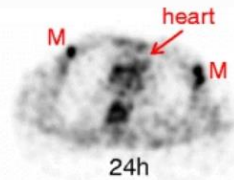

$24 \mathrm{~h}$

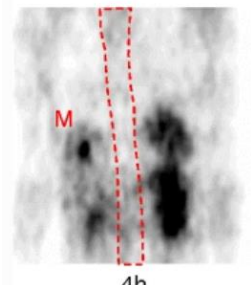

$4 \mathrm{~h}$

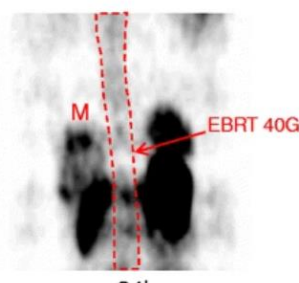

$24 \mathrm{~h}$

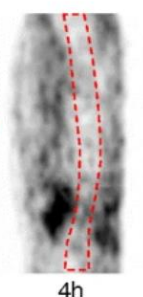

$4 \mathrm{~h}$

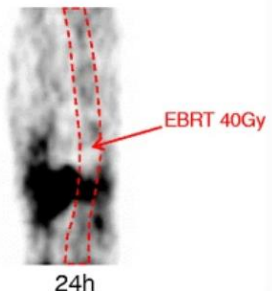

spectrometry studies evidenced that transferrin can make a ternary (or mixed) complex with the initial metal-chelator complex [32]. During the life of this ternary complex, the metal ion is transferred from the chelator to the transferrin metal binding site, likely by the Van der Waals forces. Many in vitro competition studies between transferrin and DOTA evidenced the transferrin superiority [24,33]. In vitro data using plasma from subjects injected with ${ }^{111}$ In-pentetreotide, demonstrated that after $X$ days, the radioactive metal was effectively bound to transferrin [34]

Figure 3: A: transferrin release mechanism (reprinted from [31] with permission of Springer. B: PET imaging 4 and $24 \mathrm{~h}$ post ${ }^{86} \mathrm{Y}$-DOTATOC injection from the ${ }^{86} \mathrm{Y}$-SMT-487 clinical trial. Metastases (M) already exhibit a strong uptake $4 \mathrm{~h}$ pi, while red marrow activity behaves in phase opposition to the blood pool visible in the heart. Previously irradiated vertebra by EBRT which has eradicated active marrow have a similar behavior as the blood pool. Reprinted from [24] with permission of Springer.

A recent study in human [35] showed that only $23 \%$ and $2 \%$ of ${ }^{177} \mathrm{Lu}$-DOTATATE remain intact after $24 \mathrm{~h}$ and $96 \mathrm{~h}$ postinjection, respectively. Fig. $3 \mathrm{~B}$ clearly illustrates the uptake difference between the tumor receptor-based mechanism and the transferrin transport to red marrow mechanism observed in the ${ }^{86}$ Y-SMT-487 study [24]. Directly 
during the first pass, the ${ }^{86} \mathrm{Y}$-DOTATATE binds to the tumor receptors resulting in a strong uptake within 4 hours pi, while it takes much longer time for the transferrin to catch the metal and to deliver it to the red marrow cells. The 4 and $24 \mathrm{~h}$ images proved the red marrow activity behavior in opposition phase versus the blood pool, which makes the blood-based red marrow dosimetry method unlikely to be physiologically adequate. Table 1 shows red marrow dosimetry assessments and dose-toxicity correlations reported in the recent literature.

Table 1: blood and image-based RM dose comparisons

\begin{tabular}{llll}
${ }^{177} \begin{array}{l}\text { Lu-DOTATATE } \\
\text { study }\end{array}$ & $\begin{array}{l}\text { Blood based RM } \\
\text { dosi. [mGy/MB] }\end{array}$ & $\begin{array}{l}\text { Image based RM } \\
\text { dosi. [mGy/MBq] }\end{array}$ & $\begin{array}{l}\text { toxicity-dose } \\
\text { correlation }\end{array}$ \\
\hline Santoro et al. [28] & n.a. & 0.04 [0.01-0.09] & yes \\
\hline Hagmarker et al. [29] & n.a. & $0.06[0.02-0.12]$ & yes \\
\hline Page et al. [37] & $0.02[0.01-0.03]$ & $0.06[0.03-0.11]$ & yes for imaging \\
\hline $\begin{array}{l}\text { Garsk et al. [36] } \\
\text { n.a. = not assessed }\end{array}$ & 0.016 & n.a. & no
\end{tabular}

Using 4 SPECT/CT performed after the first and second cycles, Santoro et al [28] evaluated the organs at risk in 12 patients treated with ${ }^{177} \mathrm{Lu}$-DOTATATE. The mean dosimetry for kidney and red marrow was $0.43 \pm 0.13 \mathrm{mGy} / \mathrm{MBq}$ and $0.04 \pm 0.02 \mathrm{mGy} / \mathrm{MBq}$, respectively. As the maximal tolerated dose for red marrow is about tenfold lower than that of kidney [24], this explains why hematological toxicity is the limiting factor in $26 \%$ of individually optimized ${ }^{177} \mathrm{Lu}$ PRRT [36].

Using a SPECT/CT-planar hybrid method, Hagmarker et al. [29] in ${ }^{177} \mathrm{Lu}-\mathrm{DOTATATE}$ therapy found similar red marrow dosimetry, i.e. $0.06(0.02-0.12) \mathrm{mGy} / \mathrm{MBq}$ in 22 patients without skeletal metastases. They observed a dose-platelet counts nadir relationship (fig. 4) perfectly in line with that observed in the ${ }^{90} \mathrm{Y}$-DOTATOC clinical phase I trial using ${ }^{86} \mathrm{Y}$ DOTATOC PET based red marrow dosimetry $[23,24]$.

In 200 patients treated with ${ }^{177}$ Lu-DOTATATE, Garsk et al. [36] observed fourfold lower mean blood-based red marrow dosimetry than that observed in the two reported SPECT study, i.e. $0.016 \mathrm{mGy} / \mathrm{MBq}$, which was unable to predict the hematological toxicity observed in 40 patients. Page et al. [37] compared blood based and SPECT/CT based red marrow dosimetry in 11 patients treated with ${ }^{177} \mathrm{Lu}$-DOTATATE and observed the same fourfold ratio.

The results of these six studies [28-30,35-37] urge for using post cycles SPECT/CT based red marrow dosimetry for all ${ }^{177} \mathrm{Lu}$ therapies. With optimized imaging and reconstruction methods of SPECT/CT, these studies provide evidence that direct RM uptakebased dosimetry clearly outpasses the blood-based estimations, as advocated in the

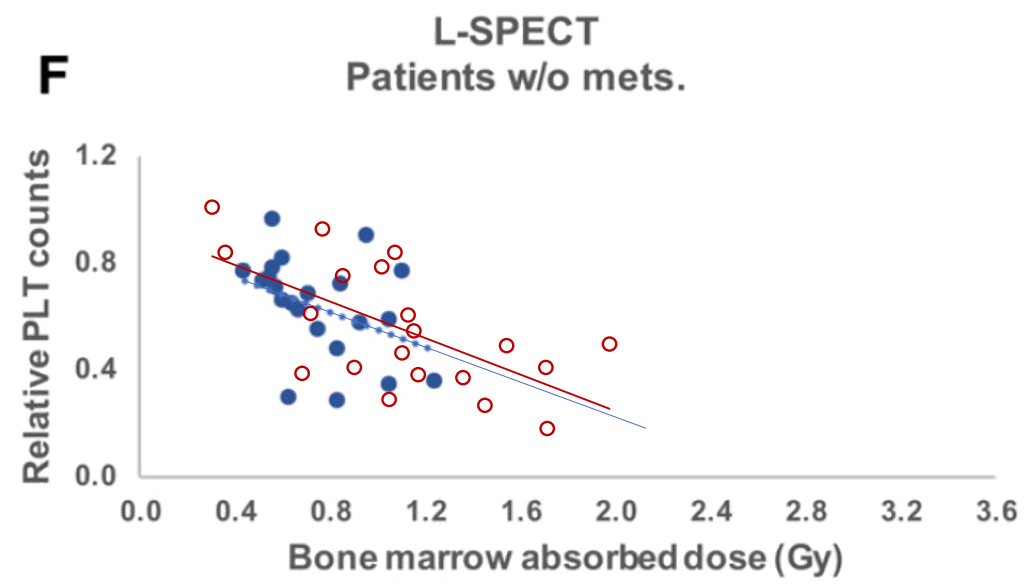


Luthatera package insert. One must keep in mind that the maximal red marrow tolerable dose is about tenfold lower than that of other tissues. Thus, a long duration, i.e., $>30$ minutes, dual-head SPECT is required to accurately assess the red marrow uptake, at several timepoints. Red marrow activity should be measured on the thoracic vertebraes: the region with the lowest attenuation and without surrounding tissues with high uptake, such as the liver, spleen or kidneys, that can produce long tail artefacts in reconstructed slices. Fortunately, nowadays, a single SPECT/CT scan encompass the thorax and the kidneys. Clearly, state-of-the art imaging capacities as available now can help improving this paradigm of RM dosimetry based on imaging, rather than on blood activity.

Figure 4: Blue solid circles: relative platelet counts decrease at nadir as a function of the hybrid SPECT/CT-planar based red marrow dose in ${ }^{177} \mathrm{Lu}$-DOTATATE therapy (reprinted from [29] with courtesy of Dr. P Bernhardt). Red empty circles: decrease observed in the ${ }^{90} \mathrm{Y}$-DOTATOC trial using ${ }^{86}$ Y-DOTATOC PET based dosimetry added by the authors [24]. Both trendlines are very similar.

\section{4. ${ }^{177} \mathrm{Lu}-90 \mathrm{Y}$ PRRT tandem therapy: a bi-orthogonal synergy}

Hobbs et al. [38] introduced the "orthogonal radionuclides" concept to promote tandem therapy with radionuclides taken up by different tissues and thus presenting different toxicities. Such tandem allows to increase the tumor dose by splitting the unwanted irradiation between different organs, as similarly performed in EBRT by rotating the beam source around the targeted tumor.

Comparing toxicities of ${ }^{177} \mathrm{Lu}$-DOTATATE and ${ }^{90} \mathrm{Y}$-DOTATOC therapies is very challenging considering the sparse informations provided in the clinical trials publications. In the Netter phase III study [10], in 116 patients receiving 4 repeated injections of 7.4GBq every 8 weeks, 0.4 and $11 \%$ underwent a renal and hematological toxicity of grade 3 or 4 , respectively. In the phase II ${ }^{90} Y$-DOTATOC study [39] in 1109 patients receiving in average 2.5 cycles of $3.7 \mathrm{GBq} / \mathrm{m} 2,9.2$ and $12.8 \%$ experienced a renal and hematological toxicity of grade 3 or 4 , respectively. However, the frequency of this hematological toxicity cannot be directly compared: indeed assuming similar uptakes, the ${ }^{90} \mathrm{Y}$ protocol delivered a much higher tissue dose than that of ${ }^{177} \mathrm{Lu}$ (table 2). The hematological to renal toxicity ratio is about twentyfold higher for the ${ }^{177} \mathrm{Lu}$-DOTATATE therapy.

Regarding dosimetry-based studies, in individualized ${ }^{177} \mathrm{Lu}$-DOTATATE study aiming to deliver $23 \mathrm{~Gy}$ to the kidney in 154 patients, $26 \%$ presented a hematological toxicity halting the therapy [36], while this event occurs only in 1 patient on 60 in the individualized ${ }^{9}$ Y-DOTATOC study aiming to deliver $27 \mathrm{~Gy}$ to the kidney [7]. Again, a twentyfold higher prevalence of hematological toxicity is observed in ${ }^{177} \mathrm{Lu}$-DOTATATE therapy.

This much higher prevalence of hematological toxicity in ${ }^{177} \mathrm{Lu}$-DOTATATE therapy is in line with the low in-vivo stability of this compound: only $23 \%$ and $2 \%$ of ${ }^{177} \mathrm{Lu}-\mathrm{DO}-$ TATATE remain intact after $24 \mathrm{~h}$ and $96 \mathrm{~h}$ post injection in patients [35] whereas $90 \%$ of ${ }^{86} \mathrm{Y}$-DOTATOC remains intact $5 \mathrm{~h}$ post injection in primates [40]. Intuitively, it makes sense that the larger electron orbital of ${ }^{177} \mathrm{Lu}$ makes it more sensitive to external Van der Waals forces coming from an open transferrin lobe. Note that, as transferrin is not excreted by the kidneys, radionuclide to protein binding analysis in urine is not appropriate to evaluate the in vivo compound stability. In vitro transchelation competition with apo-transferrin could also be biased with regard that in vivo, apo-transferrin is continuously renewed by the release of the metal ion in the red marrow. Such in vitro studies have thus to be performed with a huge apo-transferrin overload. Last, caution is also needed with in vivo rodent stability studies regarding that experiments proved different iron binding and release properties vs humans [41], that could hold true too for yttrium and lutetium.

Table 2: comparison of grade 3-4 toxicities between ${ }^{177} \mathrm{Lu}$-DOTATATE and ${ }^{90} \mathrm{Y}$ DODTATOC therapies

\begin{tabular}{|c|c|c|c|}
\hline $\begin{array}{c}{ }^{177} \mathrm{Lu}- \\
\text { DOTATATE } \\
\text { phase IUI } 101\end{array}$ & $\begin{array}{c}{ }^{90} Y- \\
\text { DOTATOC } \\
\text { phase }\end{array}$ & $\begin{array}{c}{ }^{177} \mathrm{Lu}- \\
\text { DOTATATE }\end{array}$ & $\begin{array}{c}{ }^{90} \mathrm{Y}- \\
\text { DOTATOC }\end{array}$ \\
\hline
\end{tabular}




\begin{tabular}{ccccc}
\hline & & & $\begin{array}{c}\text { indiv. optim. } \\
\text { [36] }\end{array}$ & $\begin{array}{c}\text { indiv. optim. } \\
\text { [7] }\end{array}$ \\
\hline inj. activity & $4 \times 7.4 \mathrm{GBq}$ & $\begin{array}{c}2.5 \times 10.4 \\
\mathrm{GBq} / \mathrm{m}^{2}\end{array}$ & $23 \mathrm{~Gy}$ to kidneys & $\begin{array}{c}27 \mathrm{~Gy} \text { to } \\
\text { kidneys }\end{array}$ \\
\hline $\begin{array}{c}\text { inj. decay } \\
\text { energy }\end{array}$ & $511 \mathrm{~J}$ & $2193^{*} \mathrm{~J}$ & n.a. & n.a. \\
\hline renal toxicity & 0.4 & 9.2 & n.a. & n.a. \\
\hline $\begin{array}{c}\text { hematotoxicity } \\
\text { hemato/renal } \\
\text { tox }\end{array}$ & 11.0 & 12.8 & $\mathbf{2 6 . 0}$ & $\mathbf{1 . 6}$ \\
\hline
\end{tabular}

${ }^{*}$ : assuming a body surface area of $1.7 \mathrm{~m} 2$. n.a.: not applicable. indiv.optim.: individualized optimization

All these facts clearly show that the orthogonal toxicity concept applies to the couple ${ }^{177} \mathrm{Lu}$-DOTATATE and ${ }^{90} \mathrm{Y}$-DOTATOC for which the prime tissue at risk is the red marrow and the kidney, respectively. This feature alone should be sufficient to promote the tandem approach as a new standard for PRRT therapy.

Last, but not least, this tandem is also orthogonal regarding the tumor response (justifying the bi-orthogonal appellation): by its short beta range ${ }^{177} \mathrm{Lu}$ is efficient to deliver high doses in sub-centimetric tumors, whereas the ${ }^{90} \mathrm{Y}$ beta with its one $\mathrm{cm}$ range is more efficient to cross-irradiate low vascularized regions often present in larger tumors [42,43].

This dose-response synergy was already observed in 2005 in a preclinical model [44]. Pancreatic cancer cells were successively grafted, i.e. spaced by 3 weeks, in the two opposite flanks of rats, resulting in rats bearing a small $(\approx 0.5 \mathrm{~cm} 2)$ and a large $(\approx 8 \mathrm{~cm} 2)$ tumor. Rats were split in four groups of about 12 individuals. Survival was impressively higher in rats treated with the ${ }^{177} \mathrm{Lu}$-DOTATATE \& ${ }^{90} \mathrm{Y}$-DOTATOC tandem than that of rats treated with only one radionuclide (fig. 5A).

Kunikowska et al. [45] compared overall survival (OS) between single ${ }^{90} \mathrm{Y}$-DOTATOC therapy and ${ }^{90} \mathrm{Y}-{ }^{177} \mathrm{Lu}$ tandem therapy. The patients were not randomly drawn: first 25 consecutive patients were treated with ${ }^{90} \mathrm{Y}$ alone $(7.4 \mathrm{GBq} / \mathrm{m} 2)$, and the following 25 consecutive patients were treated with the ${ }^{90} \mathrm{Y}-177 \mathrm{Lu}$ tandem $(3.7-3.7 \mathrm{GBq} / \mathrm{m} 2)$. However, the same patient enrollment criteria were chosen for both groups. Such as in the rat study, the OS was impressively better in the tandem group, especially that assuming similar uptake, the tandem delivers absorbed doses $30 \%$ lower than that of the ${ }^{90} \mathrm{Y}$ alone using this activity protocol.
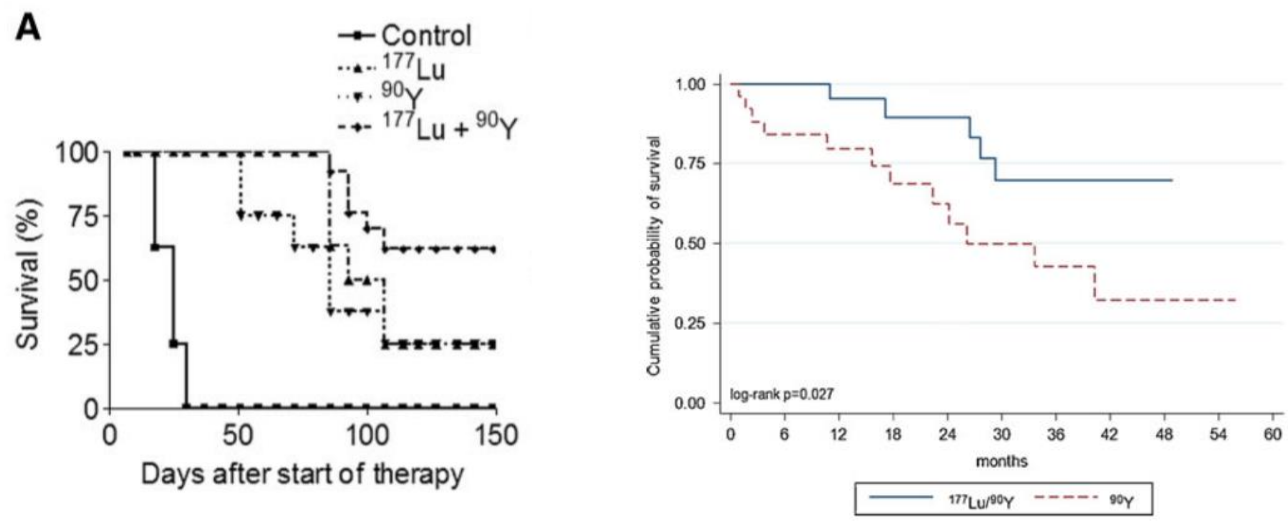

Figure 5: A: survival in rats bearing a small and large tumor treated with single or tandem PRRT. Reprinted from [44] with permission of the SNM. B: survival in patients treated with 7.4 $\mathrm{GBq} / \mathrm{m} 2$ of ${ }^{90} \mathrm{Y}$-DOTATOC or with $3.7 \mathrm{GBq} / \mathrm{m} 2$ of ${ }^{90} \mathrm{Y}$-DOTATOC $+3.7 \mathrm{GBq} / \mathrm{m} 2$ of ${ }^{177 \mathrm{Lu}-D O T A T A T E}$. 
Note that assuming similar uptake, the tandem delivers absorbed doses $30 \%$ lower than those of ${ }^{90} \mathrm{Y}$ alone. Reprinted from [45] with permission of Springer.

In this study, the tandem was given together in 2 or 3 cycles which is not the right strategy: the well vascularized large tumor region taking less radionuclide in the following cycle due to the additional ${ }^{177} \mathrm{Lu}$ irradiation eradicating the taking up cells. The best strategy is to first perform the ${ }^{90} \mathrm{Y}$ cycles to benefit from the high uptake of vascularized regions to cross irradiate the other tumor regions, then to end up with a ${ }^{177} \mathrm{Lu}$ cycle to efficiently irradiate, and hopefully eradicate small metastases [46].

\section{Individual PRTT optimization planning}

Multi-cycle PRRT is well adapted to individualized therapy planning as the first cycle delivered dose is safe for all patients. Afterwards, post cycle dosimetry can be performed to optimize the activity to be injected in the following cycles. Note that if $98 \mathrm{Ga}$ DOTATATE PET is of prime interest to select candidate for the PRRT therapy, it is useless for dosimetry estimation regarding its too short half-life. In ${ }^{90}$ Y-DOTATOC therapy, phantom and patient studies proved ${ }^{9} \mathrm{Y}$ PET imaging providing an accurate kidney dose estimation [47], while medium energy collimator SPECT/CT is well adapted for ${ }^{177} \mathrm{Lu}$-DOTATATE [16], provided the acquisition time is long enough.

PRRT dosimetry requires at least two imaging time-points, as tissue activity curves exhibit a bi-exponential asymmetric bell shape. This feature makes inaccurate proposed single time-point estimates [48], which is only for a single exponential starting from $t=0$, the model used to propose it [49]. One time point after the maximal uptake is needed, e.g., $24 \mathrm{~h}$ post injection, and another time-point somewhere around one effective half-life, i.e., 48 or $72 \mathrm{~h}$ post ${ }^{90} \mathrm{Y}$ injection and 5 to 8 days post ${ }^{177} \mathrm{Lu}$ injection.

Only the two tissues at risk have to be imaged, i.e. the thoracic vertebrae and the kidneys, which can be done with two PET/CT positions for ${ }^{90} \mathrm{Y}$ and with one single SPECT/CT position for ${ }^{177} \mathrm{Lu}$. Planar imaging, and combined planar and hybrid SPECT/CT should be avoided regarding the superimposition of the residual blood-pool activity for red marrow and of the liver and of the spleen activity for the kidneys. Any physician thinks mandatory to use state-of-the-art TOF-PET/CT to perform tumors staging or follow-up! It is time to tackle claims that short duration whole-body planar scan is sufficient to perform dosimetry. By always trying to simplify dosimetry to satisfy this claim, medical physics experts (MPE) are left to poor quality data for accurate dosimetry, which reinforces the common belief that dosimetry is useless.

Menda et al. [50] conducted a prospective post cycle renal dosimetry using ${ }^{90} \mathrm{Y}$ bremsstrahlung SPECT/CT in 25 patients having neuroendocrine tumours. A ${ }^{90} \mathrm{Y}$ TOFPET/CT [47] was used at the first time for bremsstrahlung SPECT/CT calibration purpose. The study confirmed the very high variability of inter-patient renal dosimetry as already observed using ${ }^{86}$ Y-DOTATOC PET [8], advocating the interest to individually optimize the injected ${ }^{90} \mathrm{Y}$ activity.

Almost twenty years after the individualized ${ }^{90}$ Y-SMT-487 trial [23], Del Petre et al. [51], Sundlov et al. [52] and Garske-Roman et al. [36] performed SPECT/CT based individualized planning in ${ }^{177} \mathrm{Lu}$-DOTATATE therapy in 52, 51 and 200 patients aiming at a renal $\mathrm{BED}=27 \mathrm{~Gy}, \mathrm{D}=23 \mathrm{~Gy}$ and $\mathrm{D}=23 \mathrm{~Gy}$, respectively. They demonstrated that applying the standard recommendation of four 7.4GBq-cycles results in undertreating 85, 73 and $49 \%$ of the patients.

Garske-Roman et al [36] observed an impressive progression free survival (PFS) and overall survival (OS) improvement of patients who were able to reach the 23Gy renal dose limit $(n=114)$ versus those who could not due to hematological toxicities $(n=40)$ (fig. 6). 


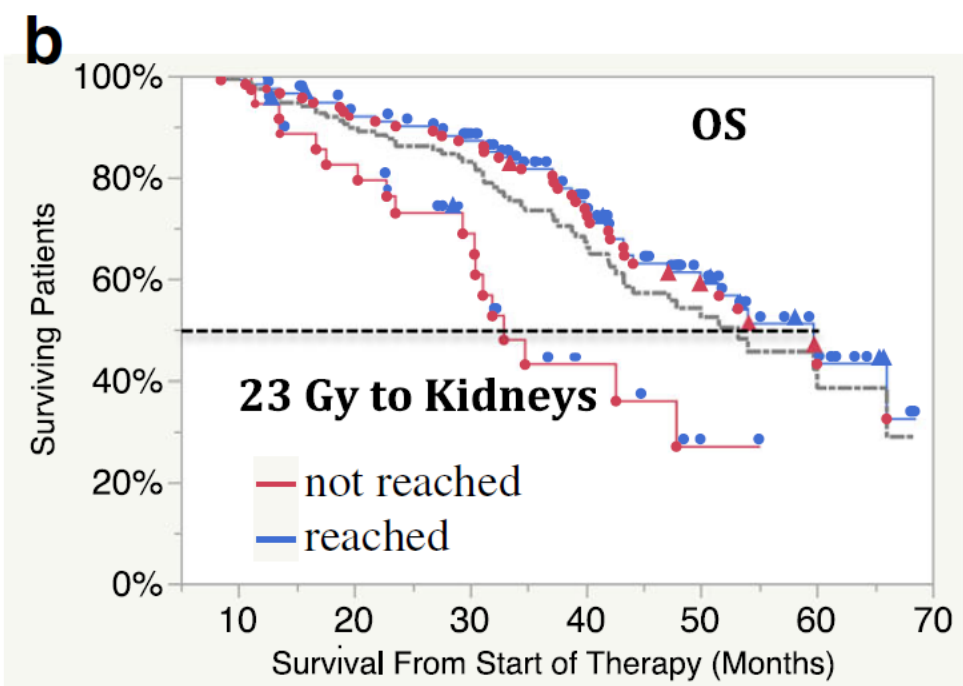

Figure 6: Overall survival in ${ }^{177} \mathrm{Lu}$-DOTA-octreotate therapy in relation to $23 \mathrm{~Gy}$ achieved to the kidney (blue curve), or not due to haematological toxicity (red curve). Grey curve: all patients OS. Red symbol: patients died. Blue symbol: patient alive. (reprinted from [36] with courtesy of Dr. Sundin).

\section{Smart optimized tandem therapy design: a proposal}

As in tandem therapy, the potential toxicity is split between two different tissues, the activity fractionation can be reduced versus a single radionuclide therapy, which has the benefit to reduce aggressive tumours regrowth between cycles. As a result, a smart full optimized tandem therapy, fractionated in three cycles, can be proposed (fig. 7). It requires only 4 patient visits to the hospital, 3 amino-acid infusions and 5 imaging sessions.

At day 0 , a fixed activity of ${ }^{90} \mathrm{Y}$-DOTATOC/TATE is injected with amino-acid infusion. The day after before releasing the patient from the hospital, a ${ }^{90} \mathrm{Y}$ kidney $30 \mathrm{~min}-$ $\mathrm{PET} / \mathrm{CT}$ is performed.

At day 3, a ${ }^{90} \mathrm{Y}$ kidneys $30 \mathrm{~min}-\mathrm{PET} / \mathrm{CT}$ is performed to compute the kidney dosimetry in order to assess the residual ${ }^{90} \mathrm{Y}$ activity needed to reach a BED of $15 \mathrm{~Gy}$ to the kidneys. Afterwards, this ${ }^{90} Y$-DOTATOC/TATE activity complement is injected to the patient together with a fixed ${ }^{177} \mathrm{Lu}$-DOTATATE activity along with amino-acid infusion, blood sample is withdrawn to obtain the cell count base line. As, this session corresponds to the last ${ }^{90} \mathrm{Y}$ cycle, co-injection of ${ }^{177} \mathrm{Lu}$ does not impact the ${ }^{90} \mathrm{Y}$ cross irradiation. Note that with regards to the effective kidney half-life ( $\approx 30 \mathrm{~h},[8])$, the initial dose rate is already reduced by a factor $\approx 5$, corresponding to an effective dose fractionation. The day after, before releasing the patient, a ${ }^{177} \mathrm{Lu}$ thorax-abdomen $30 \mathrm{~min}$ dual-head SPECT/CT is performed. Additionally, for a highly valuable scientific point of view, a blood sample could be taken to assess the relative binding of ${ }^{177} \mathrm{Lu}$ and ${ }^{90} \mathrm{Y}$ to transferrin in the same patient at the same time using size-exclusion chromatography, with appropriate MW standards.

At day 8 , a ${ }^{177} \mathrm{Lu}$ thorax-abdomen SPECT/CT is performed, and the kidney and RM dosimetry is computed. The kidney dosimetry should be computed using the Sfactors taking into account the ${ }^{90} \mathrm{Y}$ and ${ }^{177} \mathrm{Lu}$ beta range for the cross irradiation of the glomerulus by the taking up tubules [21]. At day 31, a blood sample is taken by the treating team or by the general practitioner to estimate the blood cell count nadir.

At day 41 , a blood sample is withdrawn to check the cells counts recovery, and depending on the values at nadir and recovery, a ${ }^{177} \mathrm{Lu}$-DOTATATE activity complement is injected satisfying for the whole therapy the two limits: $\mathrm{D}<2$ Gy to the red marrow and $\mathrm{BED}<31$ Gy to the kidneys. Note that the physician has the competence to modulate these 
limits according to the patient status and to his cells counts recovery. The day after, before releasing the patient a thorax-abdomen ${ }^{177} \mathrm{Lu}$ SPECT/CT is performed.

Afterwards, the patient undergoes the normal follow-up.

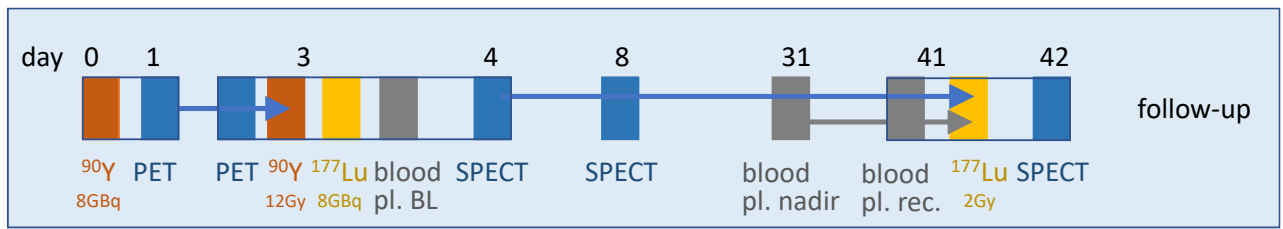

Figure 7: proposed smart optimized tandem therapy work flow including 4 patient visits to the hospital, with one or two imaging at each visit.

\section{Conclusion}

These recent years, many studies provided evidences of the huge benefit for the patient outcome to individually optimize the activity to be injected. Furthermore, although not performed along an optimized planning, recent studies have also established the importance to profit of the bi-orthogonal toxicity-response patterns of the tandem ${ }^{90} \mathrm{Y}$-DOTATOC-177Lu-DOTATATE. No doubt, that combination of these two approaches, such as proposed in fig.7, will still improve the patient outcome and will push PRRT to a real curative intent.

However, last year, the EANM published a position paper [53] on the article 56 of the Council Directive 2013/95 Euratom which required an individualized optimisation planning in all radiotherapies. This position paper aims to provide a guidance on how to interpret the Directive statements and states that ${ }^{177} \mathrm{Lu}$-DOTATATE, used according to the package insert, is a standard therapy not requiring any individualized planning, which is scientifically questionable regarding the present review.

However, let's recall that legally the MPE responsibility is framed by the corresponding national transposition of the directive. But, MPE or practitioner cannot be prosecuted for having followed the directive 2013/95 rather than the national implementation. Indeed, as the directive 2013/95 provisions are unconditional and sufficiently clear and precise, the directive has direct effects, and any individual can invoke the directive provisions in front of any national court [54]. The European Court of Justice jurisprudence has extended this principle to cases with incorrect implementation of a directive [55].

\section{Supplementary Materials: none.}

Author Contributions: Conceptualization, SW and FJ; methodology, SW and FJ.; formal analysis, SW and FJ; investigation, SW and FJ.; resources, SW and FJ.; writing-original draft preparation, SW and FJ.; writing - review and editing, SW and FJ; supervision, SW and FJ. All authors have read and agreed to the published version of the manuscript.

Funding: No funding was received.

Institutional Review Board Statement: not applicable.

Informed Consent Statement: not applicable.

Data Availability Statement: all data came for published articles.

Acknowledgments:

Conflicts of Interest: The authors declare no conflict of interest

\section{References}

1. Krenning EP, De Jong M, Kooij PP, Breeman WA, Bakker WH, De Herder WW, Van Eijck CH, Kwekkeboom DJ, Jamar F, Pauwels S, Valkema R. Radiolabelled somatostatin analogue(s) for peptide receptor scintigraphy and radionuclide therapy. Ann Oncol. 
2. Laster BH, Shani G, Kahl SB, Warkentien L. The biological effects of Auger electrons compared to $\alpha$-particles and Li ions. Acta Oncol. 1996,35,917-23.

3. De Jong M, Valkema R, Jamar F, Kvols LK, Kwekkeboom DJ, Breeman WA, Bakker WH, Smith C, Pauwels S, Krenning EP. Somatostatin receptor-targeted radionuclide therapy of tumors: preclinical and clinical findings. Sem nucl med. 2002,32,133-140.

4. Barone R, Walrand S, Konijnenberg M, Valkema R, Kvols LK, Krenning EP, Pauwels S, Jamar F. Therapy using labelled somatostatin analogues: comparison of the absorbed doses with 111In-DTPA-D-Phe1-octreotide and yttrium-labelled DOTA-DPhe1-Tyr3-octreotide. Nucl med com. 2008,29,283-90.

5. Otte A, Herrmann R, Heppeler A, Behe M, Jermann E, Powell P, Maecke HR, Muller J. Yttrium-90 DOTATOC: first clinical results. Eur J Nucl Med. 1999,26,1439-47.

6. Jamar F, Barone R, Mathieu I, Walrand S, Labar D, Carlier P, De Camps J, Schran H, Chen T, Smith MC, Bouterfa H. 86 Y-DOTA 0-d-Phe 1-Tyr 3-octreotide (SMT487) - a phase 1 clinical study: pharmacokinetics, biodistribution and renal protective effect of different regimens of amino acid co-infusion. Eur J Nucl Med Mol Imag. 2003,30,510-8.

7. Valkema R, Pauwels SA, Kvols LK, Kwekkeboom DJ, Jamar F, de Jong M, Barone R, Walrand S, Kooij PP, Bakker WH, Lasher J. Long-term follow-up of renal function after peptide receptor radiation therapy with 90Y-DOTA0, Tyr3-octreotide and 177Lu-DOTA0, Tyr3-octreotate J Nucl Med. 2005,46,83S-91S.

8. Barone R, Borson-Chazot F, Valkema R, Walrand S, Chauvin F, Gogou L, Kvols LK, Krenning EP, Jamar F, Pauwels S. Patientspecific dosimetry in predicting renal toxicity with 90Y-DOTATOC: relevance of kidney volume and dose rate in finding a doseeffect relationship. J Nucl Med. 2005,46,99S-106S.

9. Kwekkeboom DJ, Bakker WH, Kam BL, Teunissen JJ, Kooij PP, de Herder WW, et al. Treatment of patients with gastroenteropancreatic (GEP) tumours with the novel radiolabelled somatostatin analogue [177Lu-DOTA(0), Tyr(3)]octreotate. Eur J Nucl Med 2003,30,417-22.

10. Strosberg J, El-Haddad G, Wolin E, Hendifar A, Yao J, Chasen B, Mittra E, Kunz PL, Kulke MH, Jacene H, Bushnell D. Phase 3 trial of 177Lu-Dotatate for midgut neuroendocrine tumors. New Eng J Med. 2017,376,125-35.

11. Snyder WS, Ford MR, Warner GG, Watson S. MIRD pamphlet no. 11: S, absorbed dose per unit cumulated activity for selected radionuclides and organs. New York: Soc Nucl Med. 1975.

12. Walrand S, Jamar F, Mathieu I, De Camps J, Lonneux M, Sibomana M, Labar D, Michel C, Pauwels S. Quantitation in PET using isotopes emitting prompt single gammas: application to yttrium-86. Eur J Nucl Med Mol Imag. 2003,30,354-61.

13. Wessels BW, Konijnenberg MW, Dale RG, Breitz HB, Cremonesi M, Meredith RF, Green AJ, Bouchet LG, Brill AB, Bolch WE, Sgouros G. MIRD pamphlet no. 20: the effect of model assumptions on kidney dosimetry and response-implications for radionuclide therapy. J Nucl Med. 2008,49,1884-99.

14. McParland BJ. Nuclear medicine radiation dosimetry: advanced theoretical principles. Springer-Verlag London 2010, 427-430.

15. Helisch A, Förster GJ, Reber H, Buchholz HG, Arnold R, Göke B, Weber MM, Wiedenmann B, Pauwels S, Haus U, Bouterfa H. Pre-therapeutic dosimetry and biodistribution of 86 Y-DOTA-Phe 1-Tyr 3-octreotide versus 111 In-pentetreotide in patients with advanced neuroendocrine tumours. Eur J Nucl Med Mol Imag. 2004,31,1386-92.

16. Rydén T, Lagerlöf JH, Hemmingsson J, Marin I, Svensson J, Båth M, Gjertsson P, Bernhardt P. Fast GPU-based Monte Carlo code for SPECT/CT reconstructions generates improved 177 Lu images. EJNMMI phys. 2018,5,1-2.

17. Hammond PJ, Wade AF, Gwilliam ME, Peters AM, Myers MJ, Gilbey SG, Bloom SR, Calam J. Amino acid infusion blocks renal tubular uptake of an indium-labelled somatostatin analogue. Brit J cancer. 1993,67,1437-9.

18. Barone R, Pauwels S, De Camps J, Krenning EP, Kvols LK, Smith MC, Bouterfa H, Devuyst O, Jamar F. Metabolic effects of amino acid solutions infused for renal protection during therapy with radiolabelled somatostatin analogues. Nephr Dial Transp. 2004,19,2275-81.

19. Barone R, Van Der Smissen P, Devuyst O, Beaujean V, Pauwels S, Courtoy PJ, Jamar F. Endocytosis of the somatostatin analogue, octreotide, by the proximal tubule-derived opossum kidney (OK) cell line. Kidney Int. 2005,67,969-76. 
20. de Jong M, Barone R, Krenning E, Bernard B, Melis M, Visser T, Gekle M, Willnow TE, Walrand S, Jamar F, Pauwels S. Megalin is essential for renal proximal tubule reabsorption of 111In-DTPA-octreotide. J Nucl Med. 2005,46,1696-700.

21. Konijnenberg M, Melis M, Valkema R, Krenning E, De Jong M. Radiation dose distribution in human kidneys by octreotides in peptide receptor radionuclide therapy. J Nucl Med. 2007,48,134-42.

22. not yet identified,

23. Pauwels S, Barone R, Walrand S, Borson-Chazot F, Valkema R, Kvols LK, Krenning EP, Jamar F. Practical dosimetry of peptide receptor radionuclide therapy with 90Y-labeled somatostatin analogs. J Nucl Med. 2005,46,92S-8S.

24. Walrand S, Barone R, Pauwels S, Jamar F. Experimental facts supporting a red marrow uptake due to radiometal transchelation in 90 Y-DOTATOC therapy and relationship to the decrease of platelet counts. Eur J Nucl Med Mol Imag. 2011,38,1270-80.

25. Hartmann H, Oehme L, Kotzerke J. ${ }^{86}$ Y-DOTATOC uptake in red marrow is not routinely visible. Eur J Nucl Med Mol Imag. 2011,38,1384.

26. Forrer F, Krenning EP, Kooij PP, Bernard BF, Konijnenberg M, Bakker WH, Teunissen JJ, de Jong M, van Lom K, de Herder WW, Kwekkeboom DJ. Bone marrow dosimetry in peptide receptor radionuclide therapy with [177 Lu-DOTA 0, Tyr 3] octreotate. Eur J Nucl Med Mol Imag. 2009,36,1138-46.

27. https://www.ema.europa.eu/en/documents/product-information/lutathera-epar-product-information en.pdf

28. Santoro L, Mora-Ramirez E, Trauchessec D, Chouaf S, Eustache P, Pouget JP, Kotzki PO, Bardiès M, Deshayes E. Implementation of patient dosimetry in the clinical practice after targeted radiotherapy using [177 Lu-[DOTA0, Tyr3]-octreotate. Eur J Nucl Med Mol Imag. 2018,8,103.

29. Hagmarker L, Svensson J, Rydén T, van Essen M, Sundlöv A, Gleisner KS, Gjertsson P, Bernhardt P. Bone Marrow Absorbed Doses and Correlations with Hematologic Response During 177Lu-DOTATATE Treatments Are Influenced by Image-Based Dosimetry Method and Presence of Skeletal Metastases. J Nucl Med. 2019,60,1406-13.

30. Tipping J, Page E, Calvert N, Hamilton D, Cullen D, Price E, Pells S, Needham G, Manoharan P. Improved prediction of haematological toxicity during 177Lu Lu-DOTA-TATE therapy by optimising serial SPECT-CT red marrow dosimetry. Eur J Nucl Med Mol Imag. 2020,47,S239

31. Hamilton DH, Turcot I, Stintzi A, Raymond KN. Large cooperativity in the removal of iron from transferrin at physiological temperature and chloride ion concentration. J Biol Inorg Chem. 2004,9,936-44.

32. Bates GW, Billups C, Saltman P. The Kinetics and Mechanism of Iron (III) Exchange between Chelates and Transferrin II. The presentation and removal with ethylenediaminetetraacetate. J Biol Chem. 1967,242,2816-21.

33. EW price. Synthesis, evaluation, and application of new ligands for radiometal based radiopharmaceuticals. 2014. Thesis. University British Columbia. https://open.library.ubc.ca/cIRcle/collections/ubctheses/24/items/1.0103411

34. Barone R, Walrand S, Leveque P, Pauwels S, Jamar F. Red marrow toxicity after therapy with radiolabelled somatostatin analogues: a possible role of plasma protein binding. Eur J Nucl Med 2003, 30,232.

35. Lubberink M, Wilking H, Öst A, Ilan E, Sandström M, Andersson C, Fröss-Baron K, Velikyan I, Sundin A. In vivo instability of 177Lu-DOTATATE during peptide receptor radionuclide therapy. J Nucl Med. 2020,61,1337-40.

36. Garske-Roman U, Sandström M, Fröss Baron K, Lundin L, Hellman P, Welin S, Johansson S, Khan T, Lundqvist H, Eriksson B, Sundin A, Granberg D. Prospective observational study of 177Lu-DOTA-octreotate therapy in 200 patients with advanced metastasized neuroendocrine tumours (NETs): feasibility and impact of a dosimetry-guided study protocol on outcome and toxicity. Eur J Nucl Med Mol Imag. 2018,45,970-88.

37. Page E, Tipping J, Hamilton D, Cullen D, Calvert N, Pietras B, Pells S, Price E. Red marrow dosimetry for PRRT: blood versus 3D image based methods of activity measurement.

38. Hobbs RF, Wahl RL, Frey EC, Kasamon Y, Song H, Huang P, Jones RJ, Sgouros G. Radiobiologic optimization of combination radiopharmaceutical therapy applied to myeloablative treatment of non-Hodgkin lymphoma. J Nucl Med. 2013,54,1535-42. 
39. Imhof A, Brunner P, Marincek N, Briel M, Schindler C, Rasch H, Mäcke HR, Rochlitz C, Müller-Brand J, Walter MA. Response, survival, and long-term toxicity after therapy with the radiolabeled somatostatin analogue [90Y-DOTA]-TOC in metastasized neuroendocrine cancers. J Clin Oncol. 2011,29,2416-23.

40. Rösch F, Herzog H, Stolz B, Brockmann J, Köhle M, Mühlensiepen H, Marbach P, Müller-Gärtner HW. Uptake kinetics of the somatostatin receptor ligand [86 Y] DOTA-d Phe 1-Tyr 3-octreotide ([86 Y] SMT487) using positron emission tomography in nonhuman primates and calculation of radiation doses of the 90 Y-labelled analogue. Eur J Nucl Med. 1999,26,358-66.

41. Welch S, Skinner A. A comparison of the structure and properties of human, rat and rabbit serum transferrin. Comparative biochemistry and physiology. B, Comparative biochemistry. 1989,93,417-24.

42. Konijnenberg MW, de Jong M. Preclinical animal research on therapy dosimetry with dual isotopes. Eur J Nucl Med Mol Imag. 2011,38,19-27.

43. Walrand S, Hanin FX, Pauwels S, Jamar F. Tumour control probability derived from dose distribution in homogeneous and heterogeneous models: assuming similar pharmacokinetics, $125 \mathrm{Sn}-177 \mathrm{Lu}$ is superior to $90 \mathrm{Y}-177 \mathrm{Lu}$ in peptide receptor radiotherapy. Phys Med Biol. 2012,57,4263.

44. de Jong M, Breeman WA, Valkema R, Bernard BF, Krenning EP. Combination radionuclide therapy using 177Lu-and 90Y-labeled somatostatin analogs. J Nucl Med. 2005,46,13S-7S.

45. Kunikowska J, Królicki L, Hubalewska-Dydejczyk A, Mikołajczak R, Sowa-Staszczak A, Pawlak D. Clinical results of radionuclide therapy of neuroendocrine tumours with 90 Y-DOTATATE and tandem 90 Y/177 Lu-DOTATATE: which is a better therapy option? Eur J Nucl Med Mol Imag. 2011,38,1788-97.

46. Kong G, Callahan J, Hofman MS, Pattison DA, Akhurst T, Michael M, Eu P, Hicks RJ. High clinical and morphologic response using 90 Y-DOTA-octreotate sequenced with 177 Lu-DOTA-octreotate induction peptide receptor chemoradionuclide therapy (PRCRT) for bulky neuroendocrine tumours. Eur J Nucl Med Mol Imag. 2017,44,476-89.

47. Walrand S, Jamar F, van Elmbt L, Lhommel R, Bekonde EB, Pauwels S. 4-Step renal dosimetry dependent on cortex geometry applied to $90 \mathrm{Y}$ peptide receptor radiotherapy: evaluation using a fillable kidney phantom imaged by 90Y PET. J Nucl Med. 2010,51,1969-73.

48. Hou X, Brosch J, Uribe C, Desy A, Boning G, Beauregard JM, Celler A, Rahmim A. Feasibility of single-time-point dosimetry for radiopharmaceutical therapies. J Nucl Med. 2020,30,jnumed-120.

49. Sundlöv A, Gustafsson J, Brolin G, Mortensen N, Hermann R, Bernhardt P, Svensson J, Ljungberg M, Tennvall J, Gleisner KS. Feasibility of simplifying renal dosimetry in $177 \mathrm{Lu}$ peptide receptor radionuclide therapy. EJNMMI phys. 2018,5,1-9.

50. Menda Y, Madsen MT, O’Dorisio TM, Sunderland JJ, Watkins GL, Dillon JS, Mott SL, Schultz MK, Zamba GK, Bushnell DL, O’Dorisio MS. 90Y-DOTATOC Dosimetry-Based Personalized Peptide Receptor Radionuclide Therapy. J Nucl Med. 2018,59,1692-8. 51. Del Prete M, Buteau FA, Arsenault F, Saighi N, Bouchard LO, Beaulieu A, Beauregard JM. Personalized 177 Lu-octreotate peptide receptor radionuclide therapy of neuroendocrine tumours: initial results from the P-PRRT trial. Eur J Nucl Med Mol Imag. 2019,46,728-42.

52. Sundlöv A, Sjögreen-Gleisner K, Svensson J, Ljungberg M, Olsson T, Bernhardt P, Tennvall J. Individualised 177 Lu-DOTATATE treatment of neuroendocrine tumours based on kidney dosimetry. Eur J Nucl Med Mol Imag. 2017,44,1480-9.

53. Konijnenberg M, Herrmann K, Kobe C, Verburg F, Hindorf C, Hustinx R, Lassmann M. EANM position paper on article 56 of the Council Directive 2013/59/Euratom (basic safety standards) for nuclear medicine therapy. European journal of nuclear medicine and molecular imaging. 2021,48,67-72.

54. https://eur-lex.europa.eu/legal-content/EN/TXT/HTML/?uri=LEGISSUM:114547\&from=HR 55.

https://curia.europa.eu/juris/showPdf.jsf?text=\&docid=85945\&pageIndex=0\&doclang=EN\&mode=req\&dir=\&occ=first\&part=1\&cid= 771955 para. 2 